\title{
Correlation of Ki 67, Histopathological Features and Shape of Lesion in Skeletally Mature Osteochondroma
}

\author{
Wafaa Redha Mohammed Al Sabbagh', Mushtaq Talib Hussein², Ali Fadhil Hashim³, \\ Enass Abdul Kareem Dagher Al-Saadi ${ }^{1}$ \\ ${ }^{1}$ Lecturer, Department of Pathology, College of Medicine, Kerbala University, Kerbala, Iraq, ${ }^{2}$ Assistant Prof., \\ Department Of Surgery, College of Medicine, University of Babylon, Babylon, Iraq, ${ }^{3}$ Lecturer, Department of \\ Pathology, College Of Dentistry, Kerbala University, Kerbala, Iraq
}

\begin{abstract}
Background: Osteochondroma represents the most common benign tumor of bones and accounts for about $30-50 \%$ of them. poor information's in previous studies act on demonstration of the significance of atypical histological changes as a premalignant precursor in mature osteochondroma regardless of the cap thickness. In this study, the proliferative activity of cartilaginous cap will be studied in skeletally mature patients regardless of the cap thickness to demonstrate the independent significant of histological features in predicting the malignant potential in osteochondroma. methodology : cases of skeletally mature osteochondroma (78 case) were isolated and separated into sessile and pedunculated, then into G1(without suspicious histological features) and G2 ( with suspicious features), and stained with Ki- 67 . Data were analyzed using SPSS version 21 and $\mathrm{p}$ value $<0.05$ was considered as significant. Results: There was significant association between study groups and $\mathrm{Ki} 67$ staining results, majority (75\%) of patients with suspicious histopathological features presented with positive results of Ki 67 staining ( $\mathrm{p}$ value $<0.001$ ). while there was no correlation between shape of lesion and histopathological features ( $p$ value 0.662 ). Conclusion: atypical histopathological features can be regarded as independent factor with the other clinical data when considering the diagnosis of grade 1 chondrosarcoma.
\end{abstract}

Key words: osteochondroma, proliferative marker, atypical histopathology, lesion shape, Ki 67 .

\section{Introduction}

Osteochondroma represents the most common benign tumor of the bony skeleton and accounts for about $30-50 \%$ of them. ${ }^{(1-2)}$ some researchers regards this tumor as developmental abnormality rather than a true neoplasm. It may result from small fracture in the growth plate in skeletally immature bone and herniated through periosteal bone to form a tumor like lesion. ${ }^{(3-4)}$ pathologically, osteochondroma ( also called exostosis) is composed of cartilaginous cap of hyaline type , covered with fibrous sheath that is continuous with the periosteum and it in turn covering an underlying bone and medullary cavity that is continuous with that of the parent bone ${ }^{(5-6)}$ this continuity with the underlying bone is important radiological feature in the diagnosis and it is more clear in pedunuculated shape lesion than in sessile one. Development of osteochondroma in children whom treated with radiation for Wilm's tumor due to separation and migration of physeal plate support the suggestion that osteochondroma is a tumor like condition ${ }^{(7-8-9)}$, on the other hand, presence of hereditary form of osteochondroma ( hereditary multiple osteochondromatosis or called hereditary multiple exostosis HME) support the fact that osteochondroma is a true neoplasm. ${ }^{(10-11)}$ HME comprise about $15 \%$ of osteochondroma and caused by mutation in EXT1 or EXT2 genes that are located on chromosome 8 and 11 respectively (12) the most common location for osteochondroma is the metaphysis of long bones especially the lower limbs, flat bones are less commonly affected. (13) this benign tumor usually affect children and adolescent and stop growing after skeletal maturity. Clinically, it may be silent or cause disfigurement, pain due to pressure on a nerve or blood vessel and fracture $^{(14)}$ the serious complication of this neoplasm is 
malignant transformation, usually in form of secondary peripheral chondrosarcoma. Malignancy can complicate about $25 \%$ of HME and only $1-8 \%$ of solitary exostosis (15-16). At microscopy, the tumor composed of hyaline cartilage with lobulation, the chondrocytes are bland looking, evenly spaced and show ordered maturation in columns. The cartilage merges with the underlying bone that project from the bone of origin and continuous with it. Atypical histological features such as mild increased cellularity, occasional binucleation, more than one cell in the lacuna, and foci of necrosis are considered to be reactive changes in the growing cartilaginous cap and should not be overdiagnosed when the cap thickness is within usual ${ }^{(3-17)}$ the allowed cap thickness is up to $3 \mathrm{~cm}$ in children with immature skeleton and only few millimeters in skeletally mature adult ${ }^{(3-18)}$ Also new increased growth of exostosis in adult should raise the suspicion of malignant transformation into peripheral chondrosarcoma since osteochondroma is unusual to enlarge after maturity ${ }^{(19)}$. Another features that should brought the attention is the proximal location of tumor such as around the pelvis or shoulder, grossly irregular surface, cystic changes and soft tissue invasion.

Chondosarcoma arising in osteochondroma is usually of low grade as the trabecular bone permeation is usually absent, in addition, the cytological features may be bland with the exception of loss of columnar arrangement of the chondrocytes. This laid a hard work on the pathologist and necessitate clinico-radiologicalpathological correlation for such a diagnosis.(19) Eefting et al suggested a statistically significant pathological criteria that could distinguished low grade ( G 1) chondrosarcoma from proximally located benign cartilaginous tumors ${ }^{(20) .}$ Such a histological features included increased cellularity, nuclear pleomorhism, mitosis, entrapment, mucoid changes and loss of normal cellular arrangement. These features are added to the clinical data including age of patient above 40 years, proximal location and radiological data

Few studies work on demonstration of proliferative activity of osteochondroma cartilage in children comparing with proliferative activity in premature growth plate and in adult using immunohistochemistry with Ki 67 antibodies $^{(21)}$. Ki 67 is a nuclear non histone protein that is expressed in all phases of cell cycle with exception of G0.(22) most articles focused on cartilage cap thickness as the main indicator of malignant transformation. However no enough previous studies act on demonstration of the significance of atypical histological changes as a premalignant precursor in mature osteochondroma regardless of the cap thickness. In this study, the proliferative activity of cartilaginous cap of osteochondroma will be studied in skeletally mature patients regardless of the cap thickness in order to demonstrate the independent significant of atypical histological features in predicting the malignant potential in osteochondroma. Also there will be a focus on the relashionship between unusual histological features in osteochondroma( increased cellularity, occasional binucleation and mild pleomorphism) with shape of the lesion ( sessile or pedunculated ).

\section{Methodology}

Part of cases for study are collected from Al Hilla Teaching Hospital and Al-Hayat hospital retrospectively from the last 4 years ( 2015-2018). Cases with insufficient clinic-radiological data are ignored. The other part is selected prospectively from the work of orthopedic author ( use of suitable longitudinal incision to expose the tumor, careful dissection with radical excision of osteochondroma from its base with the mother bone with its basal cortex to avoid recurrence) . Exclusion criteria: osteochondroma from skeletally immature cases were excluded.

Inclusion criteria: all skeletally mature osteochondroma regardless of cartilage cap thickness were included.

Ethical issues: no patients identity or identifying photos are included in the study, so the permission of the institute, where the data are collected (including radiological and clinical information, as well as histological slides and paraffin blocks), is taken. The total number of collected cases was 130. Orthopedic author reviewed the radiological picture for each case and separate them into skeletally immature cases (excluded from the study) and skeletally mature cases (included), so the final number of cases was 78 case. Also the included cases were divided into sessile shape lesions and pedunculated shape lesions. All study's cases were reviewed by two pathologists blindly from each other and without any clinical information. Cases are separated into group 1 (conventional osteochondroma 
with no atypical histological features) and group 2 (osteochondroma with atypical histology).

Atypical histology defined by presence of increased cellularity, binucleated chondrocytes, occasional more than one cell in the lacuna, and mild pleomorphism with absence of other features that raise the suspicion of chondrosarcoma such as irregular arrangement of chondrocytes( not in ordered columns), mitosis, marked pleomorphism, necrosis, permeation and marked myxoid changes and separation of cells into specific lobules by fibrous bands.
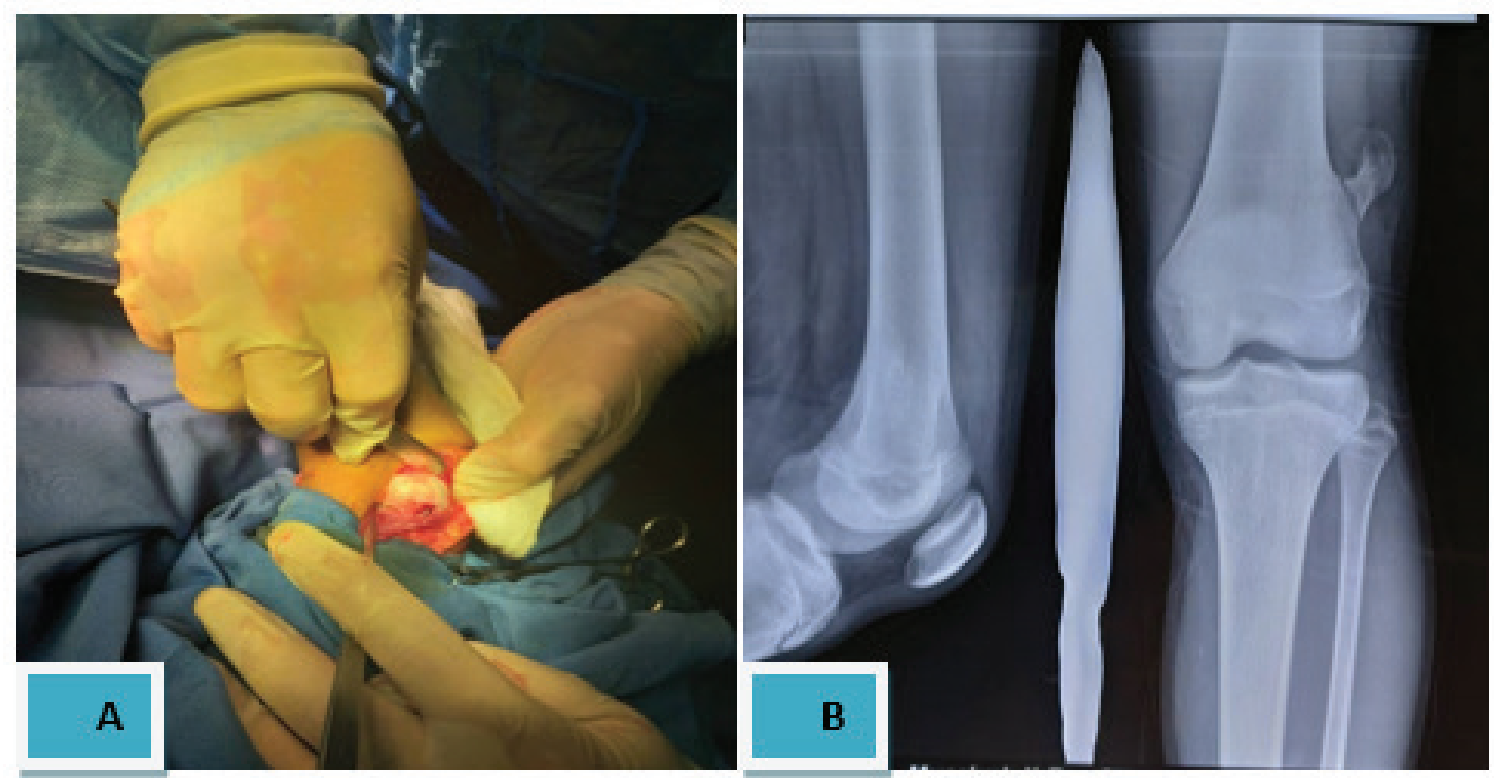

Figure (1) : A an intra-operative picture of osteochondroma in 20 years old male show pedunculated shape tumor. B X-ray of 32 years old patient show osteochondroma in skeletally mature skeleton at lower femur and complicated by basal fracture. (our cases).

\section{Data Analysis}

Statistical analysis was carried out using SPSS version 21. Categorical variables were presented as frequencies and percentages. Pearson's chi square $\left(\mathrm{X}^{2}\right)$ was used to find the association between categorical variables. A $p$-value of $\leq 0.05$ was considered as significant.

\section{Results}

The Distribution of Patients According to Study Groups
Cases of both groups are then stained immunohistochemically with MIB 1 antibody (mouse monoclonal antibody, Dako 1:50). Three $\mu \mathrm{m}$ sections are cut, deparaffinized, rehydrated and stained with standard procedure. Heat induced antigen retrieval is used for all sections. Nuclear staining of Ki 67 was scored using the average method (22) by manual counting of Ki67 positive cells in three representative sections for each case and calculating the average percentage of positive cells by two pathologists. 


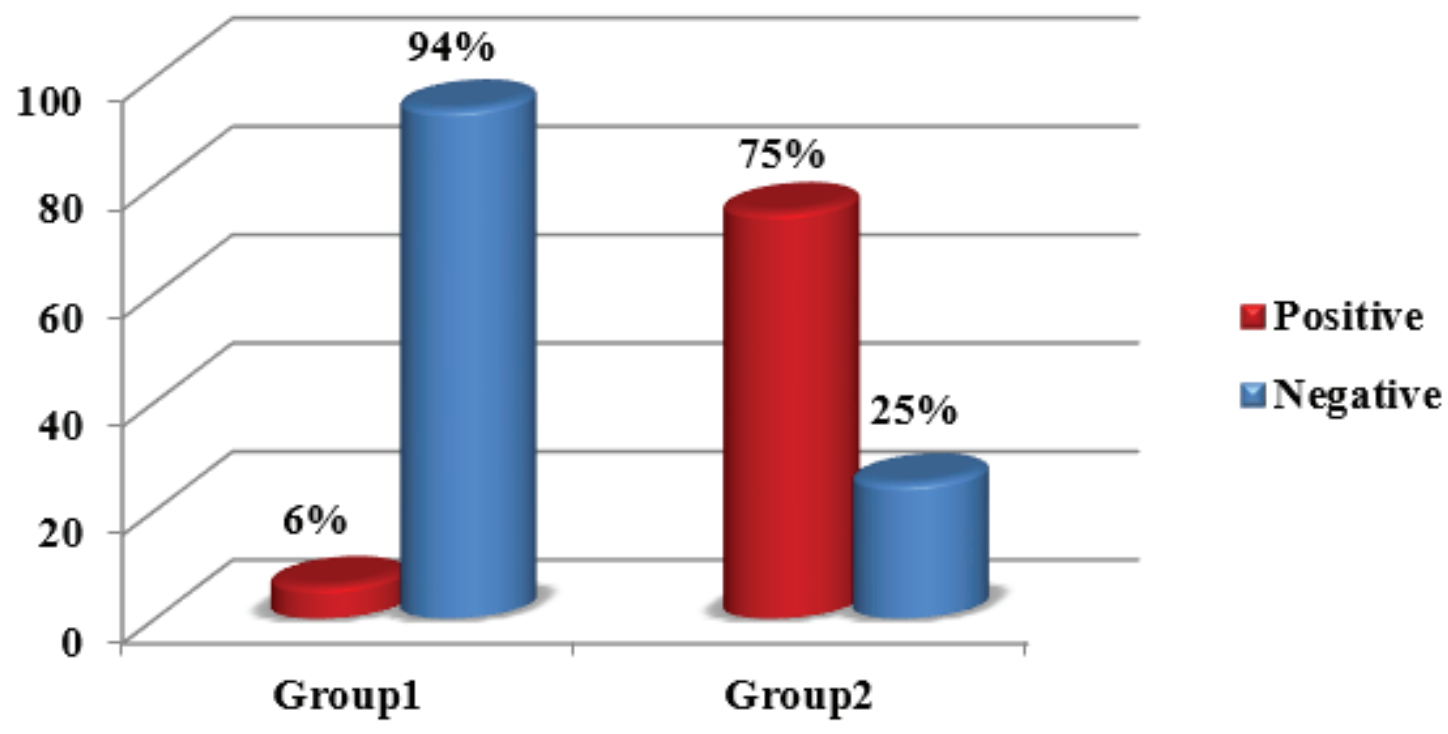

Figure 2: Distribution of patients according to study groups

The distribution of study groups according to Ki 67 staining results

Figure (3) shows distribution of study groups according to Ki 67 staining results including (Positive and negative). Majority (75\%) of patients with osteochondroma with suspicious histopathological features had positive Ki 67 staining.
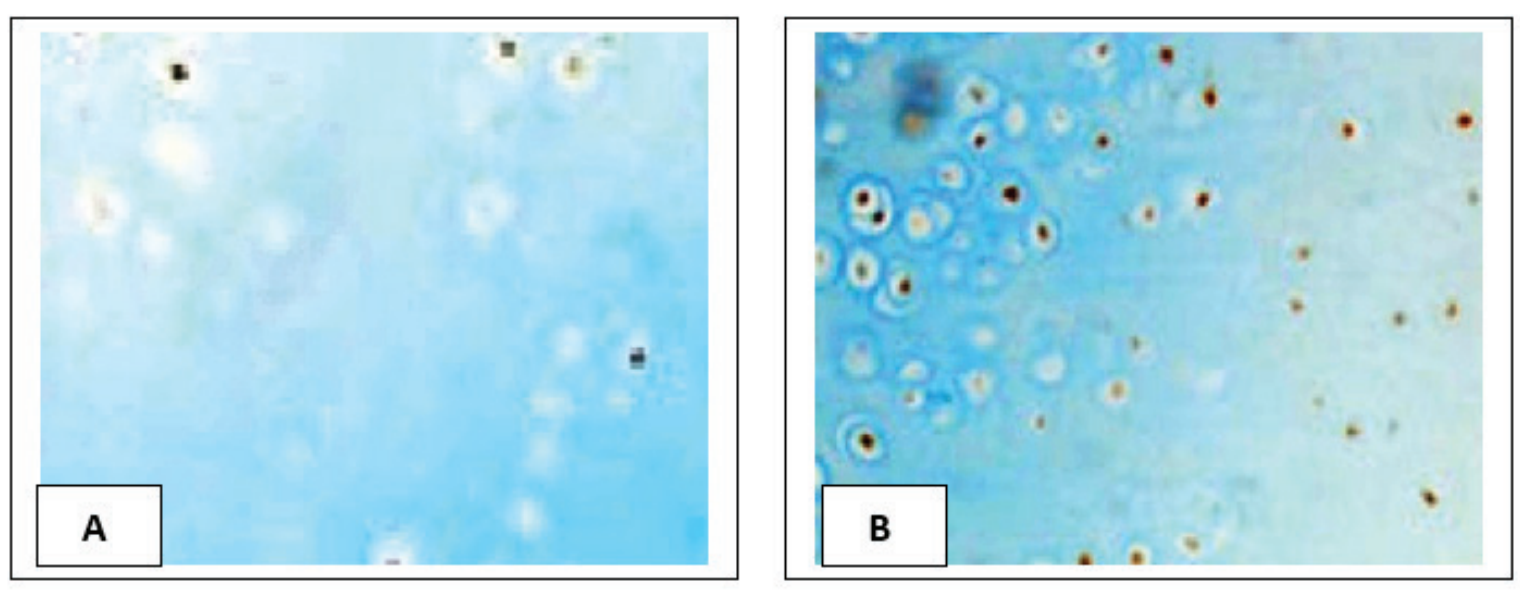

Figure 3: Distribution of study groups according to Ki 67 staining results

Figure( 4 ): A osteochondroma in 25 years male with conventional histological features (group 1) show negative Ki 67 staining. B osteochondroma in 32 years old male with increased cellularity and occasional binucleation (group 2), show positive Ki 67 staining. 


\section{The Distribution of Study Groups According to Shape of Lesion}

Figure (5) shows distribution of study groups according to shape of lesion including (pedunculated and sessile). More than a half of patients with osteochondroma in two groups had pedunculated shape of the lesion.

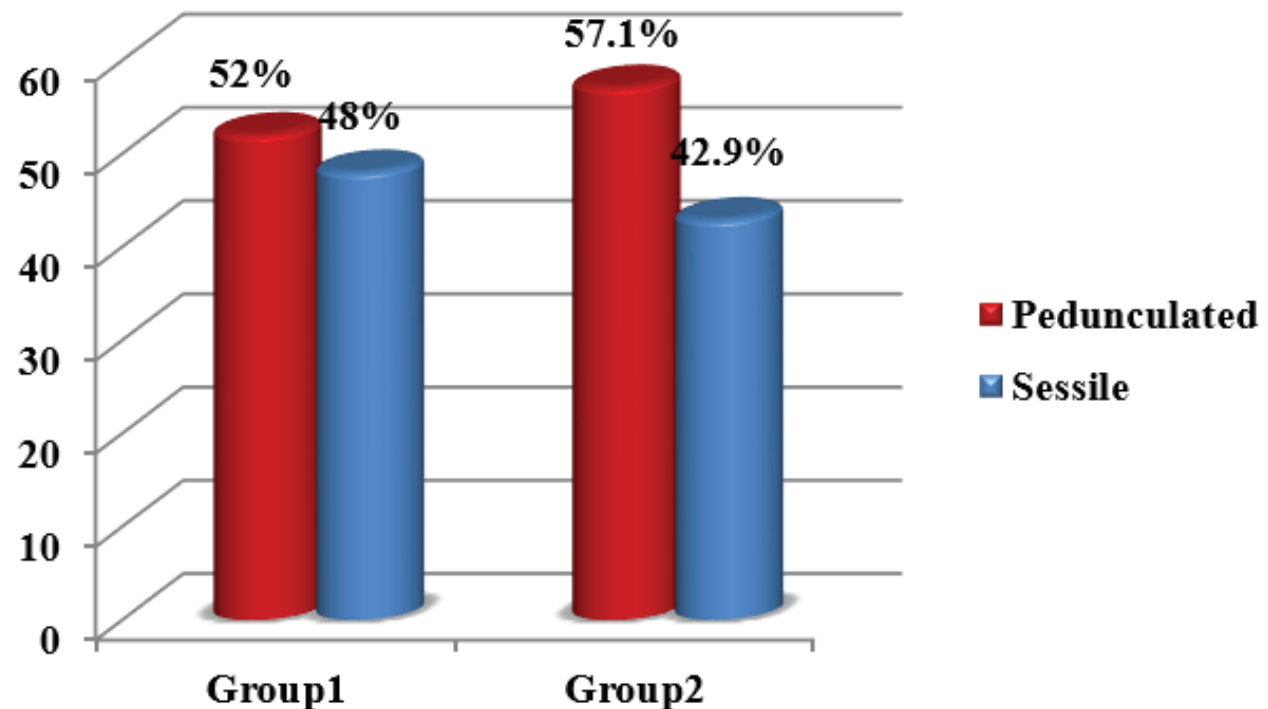

Figure 5 : Distribution of patients according to shape of lesion

The association between study groups and study variables

Table (1) shows the association between study groups including (group 1 osteochondroma without suspicious histopathological features and Group 2 osteochondroma with suspicious histopathological features), and study variables including (Ki 67 staining results and shape of the lesion).There was significant association between study groups and Ki 67 staining results, majority (75\%) of patients with suspicious histopathological features presented with positive results of Ki 67 staining ( $\mathrm{p}$ value $<0.001)$. while there was no correlation between shape of lesion and histopathological features ( $p$ value 0.662 ).

Table (1) Association between study groups and study variables including ( Ki 67 stain results and shape of lesion)

\begin{tabular}{|c|c|c|c|c|c|}
\hline \multirow{2}{*}{ Study variables } & \multicolumn{2}{|c|}{ Study groups } & \multirow{2}{*}{ Total } & \multirow{2}{*}{$\chi^{2}$} & \multirow{2}{*}{ P-value } \\
\hline & Group 1 & Group2 & & & \\
\hline $\begin{array}{l}\text { Ki } 67 \text { staining } \\
\text { Positive } \\
\text { Negative } \\
\text { Total }\end{array}$ & $\begin{array}{l}3(6.0) \\
47(94.0) \\
50(100.0)\end{array}$ & $\begin{array}{l}21(75.0) \\
7(25.0) \\
28(100.0)\end{array}$ & $\begin{array}{l}24(30.8) \\
54(69.2) \\
78(100.0)\end{array}$ & 40.11 & $<0.001 *$ \\
\hline $\begin{array}{l}\text { Shape of lesion } \\
\text { Pedunculated } \\
\text { Sessile } \\
\text { Total }\end{array}$ & $\begin{array}{l}26(52.0) \\
24(48.0) \\
50(100.0)\end{array}$ & $\begin{array}{l}16(57.1) \\
12(42.9) \\
28(100.0)\end{array}$ & $\begin{array}{l}42(53.8) \\
36(46.2) \\
78(100.0)\end{array}$ & 0.191 & 0.662 \\
\hline
\end{tabular}

*P value $\leq 0.05$ was significant. (group 1 osteochondroma without suspicious histopathological features, Group 2 osteochondroma with suspicious histopathological features). 


\section{Discussion}

In a large studies reported by Mayo Clinic, it had been found that $(81 \%)$ of secondary chondrosarcoma arose at site of previous osteochondroma ${ }^{(23) .}$ It has been estimated that $2 / 3$ of these cases derived from sporadic osteochondroma. Although osteochondroma is rarely (1$2 \%$ ) transformed into chondrosarcoma, its recognition is of high importance because most of these tumors are low grade and treated by adequate excision without need for chemotherapy or radiotherapy.(24) Huch et al performed a study on a group of solitary osteochondroma and other group of patients with multiple osteochondroma, comparing the immunohistochemical expression of Ki67 in both groups as a marker of nuclear proliferative activity. They found that the proliferative activity in osteochondroma from patients younger than 14 years of age is similar to that in postnatal growth plate, while in individuals older than 14 years there was no significant detectable proliferative activity (21). So, in the current study the only skeletally mature cases are included to avoid such overlap in the results of immunohistochemical staining with Ki-67. In a study of de Andera et al, only cellular pleomorphism and mitotic activity show significant difference between osteochondroma and low grade chondrosarcoma on one hand and high grade chondrosarcoma on the other hand. While other cellular features as binucleation, necrosis, and irregular calcification was found in $72 \%$ of osteochondroma.

K. Huch et al supported the concept of increased suspicion for malignant transformation in osteochondroma of adult when $\mathrm{Ki} 67$ positivity is detected (21)

The shape of osteochondroma ( pedunculated versus sessile ) was not studied previously regarding their relation to the microscopical features that raise the suspicion about malignancy ( increased cellularity, frequent binucleation, nodularity and necrosis). We found that no relation between the shape of the lesion and such a histological features. However the shape of the lesion may affect the accuracy of radiological diagnosis of osteochondroma ( more difficult in sessile tumors) as mentioned previously by Mark D. Murphey (2)

\section{Conclusion}

Histopathological features, when atypical, can be regarded as independent alarming factor for malignant transformation ( the same as cartilage cap thickness and may precede it). There is no relation between shape of osteochondroma and risk of atypical histological features, proliferation, and hence risk of malignancy.

Acknowledgment : We are grateful for lab staff in Al- Hilla Teaching Hospital and Al- Hayat hospital for their help in data collection.

Ethical Clearance: The Research Ethical Committee at scientific research by ethical approval of both environmental and health and higher education and scientific research ministries in Iraq

Conflict of Interest: The authors declare that they have no conflict of interest.

Funding: Self-funding

\section{References}

1. Saglik Y, Altay M, Unai VS, Basari $\mathrm{K}$ and Yildiz Y:Manifestations and management of osteochondromas: a retrospective analysis of 382 patients. Acta Orthop Belg 2006. 72:748-755,

2. Mark D. Murphey, James J. Choi, Mark J. Kransdorf, Donald J. Flemming, Frances H. Gannon: Imaging of Osteochondroma: Variants and Complications with Radiologic-Pathologic Correlation. AFIP Archives 2000. 20:1407-1434,

3. Mirra JM. Benign cartilaginous exostoses: osteochondroma and osteochondromatosis. In: Mirra JM, ed. Bone tumors: clinical, radiologic, and pathologic correlations. Philadelphia, Pa: Lea \& Febiger 1989. 2: pp.1626-1659,

4. Keith A. Studies in the anatomical changes which accompany certain growth disorders of the human body. J Anat 1920. 23: pp . 54:101,

5. Khurana J, Abdul-Karim F and Bovée JVMG: Osteochondroma. In: World Health Organization Classification of Tumours. Pathology and Genetics of Tumours of Soft Tissue and Bone. Fletcher CDM, Unni KK and Mertens F (eds.). Lyon, France: IARC 2002. pp. 234-236,

6. Bovée JVMG and Hogendoorn PCW:Multiple Osteochondromas. In: World Health Organization Classification of Tumours. Pathology and Genetics of Tumours of Soft Tissue and Bone. Fletcher 
CDM, Unni KK and Mertens F (eds.). Lyon, France, IARC 2002. pp. 360-362,

7. Langenskiöld A, Edgren W. Initiation of chondrodysplasia by localized roentgen ray injury: an experimental study of bone growth. Acta Chir Scand 1950. 99:353-373,

8. Tigges S, Nance EP. Skeletal case of the day: radiation- induced osteochondroma. AJR Am J Roentgenol 1992. 158:1368-1369,

9. Jaffe N, Ried HL, Cohen M, McNeese MD, Sullivan MP. Radiation induced osteochondroma in long-term survivors of childhood cancer. Int $\mathrm{J}$ Radiat Oncol Biol Phys 1983. 9:665-670,

10. Peterson HA. Multiple hereditary osteochondromata. Clin Orthop 1989. 239:222-230,

11. Legeai-Mallet L, Munnich A, Maroteaux P, Le Merrer M. Incomplete penetrance and expressivity skewing in hereditary multiple exostoses. Clin Genet 52:12-16, 1997.

12. Hecht JT, Hogue D, Strong LC, Hansen MF, Blanton SH, Wagner M. Hereditary multiple exostosis and chondrosarcoma: linkage to chromosome II and loss of heterozygosity for EXT-linked markers on chromosomes II and 8. Am J HumGenet. 1995. 56(5):1125-31,

13. Stieber JR, Dormans JP. Manifestations of hereditary multiple exostoses. J Am Acad Orthop Surg. 2005 13(2):110-20,

14. Karasick D, Schweitzer ME, Eschelman DJ. Symptomatic osteochondromas: imaging features. AJR Am J Roentgenol . 1997. 168:1507-1512,

15. Ahmed AR, Tan TS, Unni KK, Collins MS, Wenger DE, Sim FH. Secondary chondrosarcoma in osteochondroma: report of 107 patients. Clin
Orthop 2003. 411:193-206,

16. Dorfman HD, Czerniak B. Bone tumors. St. Louis: Mosby; 1998. p. ix.

17. Aigner $\mathrm{T}$, Frischholz $\mathrm{S}$, Dertinger $\mathrm{S}$, Beier $\mathrm{F}$, Girkontaite I, von der Mark K. Type X collagen expression and hypertrophic differentiation in chondrogenic neoplasias. Histochem Cell Biol . 1997. 107:435-440,

18. Resnick D, Kyriakos M, Greenway GD. Osteochondroma. In: Resnick D, ed. Diagnosis of bone and joint disorders. 3rd ed. Vol 5. Philadelphia, Pa: Saunders, 1995. 3725-3746,

19. A. Horvai and K.K. Unni: Premalignant conditions of bone. J Orthop Sci. 2006. 11:412-423,

20. Efting D., Schrage YM, Geirnaerdt MJ, et al. Assessment of interobserver variability and histologic parameters to improve reliability in classification and grading of central cartilaginous tumors. Am J surge Pathol . 2009. 33(1):50-7,

21. K. Huch, V. Mordstein2, J. Stöve, A.G. Nerlich3, H. Arnholdt, G. Delling, W. Puhl, et al. Expression of collagen type I, II, X and Ki-67 in osteochondroma compared to human growth plate cartilage. Eur. J. Histochem. 2002. 46: 249-258,

22. Lopez F, Belloc F, Lacombe F, Dumain P, Reiffers J, Bernard P, et al. Modalities of synthesis of Ki67 antigen during the stimulation of lymphocytes. Cytometry. 1991. 12(1):42 \pm 9 ,

23. Horvai A and Unni KK: Premalignant conditions of bone. J Orthop Sci 2006. 11: 412-423,

24. Ahmed AR, Tan TS, Unni KK, Collins MS, Wenger DE, Sim FH: Secondary chondrosarcoma: report of 107 patients. Clin Orthop 2003. 411: 193206, 